\title{
A Search for Resonant Structures in the Zodiacal Cloud with COBE DIRBE: The Mars Wake and Jupiter's Trojan Clouds
}

\author{
Marc J. Kuchner \\ Palomar Observatory, California Institute of Technology, Pasadena, CA 91125 \\ William T. Reach \\ Infrared Processing and Analysis Center, Caltech, Pasadena, CA 91125 \\ Michael E. Brown ${ }^{1}$ \\ Division of Geological and Planetary Sciences, California Institute of Technology, \\ Pasadena, CA 91125
}

Running head: Resonant Structures in the Zodiacal Cloud with DIRBE

\begin{abstract}
We searched the COBE DIRBE Sky and Zodi Atlas for a wake of dust trailing Mars and for Trojan dust near Jupiter's L5 Lagrange point. We compare the DIRBE images to a model Mars wake based on the empirical model of the Earth's wake as seen by the DIRBE and place a $3-\sigma$ upper limit on the fractional overdensity of particles in the Mars wake of $18 \%$ of the fractional overdensity trailing the Earth. We place a 3- $\sigma$ upper limit on the effective emitting area of large (10-100 micron diameter) particles trapped at Jupiter's L5 Lagrange point of $6 \times 10^{17} \mathrm{~cm}^{2}$, assuming that these large dust grains are distributed in space like the Trojan asteroids. We would have detected the Mars wake if the surface area of dust in the wake scaled simply as the mass of the planet times the Poynting-Robertson time scale.
\end{abstract}

Subject headings: asteroids - extrasolar planets - interplanetary dust - Jupiter - Mars - resonances

\footnotetext{
${ }^{1}$ Alfred P. Sloan Research Fellow
} 


\section{Introduction}

A planet interacting with a circumstellar dust cloud can produce a variety of dynamical structures in the dust. Planets can clear central holes and create large-scale asymmetries, such as arcs and warps (Roques et al. 1994). Planets can also detain dust in mean motion resonances, forming structures such as the circumsolar ring and wake of dust trailing the Earth in its orbit (Jackson and Zook 1989; Dermott et al. 1994) and clouds of dust at the planet's Lagrange points (Liou and Zook 1995).

Understanding the structures of circumstellar debris disks is vital to the search for extra-solar analogs of our solar system. Concentrations in circumstellar dust clouds may confuse planet-finding interferometers like the Keck Interferometer or the proposed Terrestrial Planet Finder (Beichman 1999). Smooth exo-zodiacal clouds can be identified by their symmetry and subtracted from the signal of a Bracewell interferometer (MacPhie and Bracewell 1979), but cloud asymmetries can be difficult to distinguish from planets (Beichman 1998). On the other hand, planet-induced asymmetries can serve to reveal the presence of a planet that is otherwise undetectable (Wyatt et al. 1999).

If we understand the inhomogeneities in our own zodiacal dust cloud, we will be better prepared to interpret observations of other planetary systems. The Diffuse Infrared Background Experiment (DIRBE) aboard the Cosmic Background Explorer (COBE) satellite has provided detailed, revealing images of the zodiacal cloud (eg. Spiesman et al. 1995, Reach et al. 1997). It surveyed the entire sky from near-Earth orbit in 10 broad infrared bands simultaneously with a $0.7^{\circ}$ by $0.7^{\circ}$ field of view over a period of 41 weeks (Boggess et al. 1992), and imaged the Earth's ring and wake (Reach et al. 1995). We investigated the COBE DIRBE data set as a source of information about structure in the solar zodiacal cloud associated with planets other than Earth.

\section{The Data Set}

We worked with a version of the DIRBE data set which contains the sky brightness with a model for the background zodiacal emission subtracted: the zodi-subtracted weekly data set from the DIRBE Sky and Zodi Atlas (DSZA). The DIRBE team created the DZSA by fitting an 88-parameter model of the zodiacal dust emission to the observed sky brightness (Kelsall et al. 1998). The model includes a smooth widened-fan component, three pairs of dust bands near the ecliptic plane, and the Earth's ring and trailing wake, but no structures associated with Mars, Jupiter, or any other planets. The zodi-subtracted weekly data set contains 41 files for each DIRBE band, spanning a period from 10 December 
1989 to 21 September 1990, covering 10 bands, centered at 1.25, 2.2, 3.5, 4.9, 12, 25, 60, 100, 140, and 240 microns.

Galactic emission dominates the zodi-subtracted maps in the mid and far-infrared near the galactic plane. Near the ecliptic plane, the zodi-subtracted maps are dominated by residuals from the subtraction of the dust bands that are associated with prominent asteroid families (Reach et al. 1997; Kelsall et al. 1998). The presence of these bands makes searching for smooth, faint heliocentric rings of dust near the ecliptic plane impossible. However, we could hope to distinguish a blob of dust following a planet across the sky from other cloud components and from the galactic background by the apparent motion of the blob during the COBE mission.

The COBE satellite orbited the Earth near the day/night terminator and repeatedly mapped a swath of the sky extending about 30 degrees before and behind the terminator (see the COBE DIRBE Explanatory Supplement (1997) for details). Each weekly map contains a robust average of all the week's data and covers a region a little larger than the daily viewing swath. This weekly averaging tends to exclude transient events that would contaminate our final maps, but should not otherwise significantly affect a search for large features that move only a few degrees per week. Figure 1 is a schematic view of the solar system during week 34 of the mission (9-16 July 1990) showing the positions of Earth, Mars and Jupiter, and the DIRBE viewing swath for that week.

Because DIRBE never imaged the sky within $60^{\circ}$ of the sun, the orbits of Mercury and Venus, for instance, do not appear in the data. Mars appeared in the DIRBE viewing swath for 25 weeks of the mission, and moved $111^{\circ}$ in ecliptic longitude during those weeks. Jupiter moved only $40^{\circ}$ in ecliptic longitude during the entire mission, but this is sufficient to allow some crude background subtraction. More distant planets moved less. Based on these constraints, we decided to search the weekly maps for dust features following the orbital paths of Mars and Jupiter. Figure 2 shows the intersection of the DIRBE viewing swath with the ecliptic plane throughout the 41 weeks of the mission, and the ecliptic longitudes of Mars, Jupiter and the Sun during those weeks.

\section{The Mars Wake}

A ring of zodiacal dust particles detained in near-Earth resonances follows the Earth around the sun (Jackson and Zook 1989; Dermott et al. 1994). This ring consists mainly of dust in mean-motion resonances where the particles orbit the sun $j$ times every

$j+1$ Earth years ( $\mathrm{j}$ is a whole number). Smaller trapped particles experience greater 
Poynting-Robertson acceleration, so the equilibrium locations of their orbital pericenters shift closer to the Earth on the trailing side, where the component of Earth's gravity that opposes Poynting-Robertson drag is stronger. The result, averaged over many particles, appears as a density enhancement in the ring behind the Earth - a trailing dust wake. The Earth's wake was detected by by IRAS (Dermott et al. 1988, Reach 1991), and later, by DIRBE as an asymmetry in the near-Earth dust brightness of $\sim 1.1 \mathrm{MJy}$ ster $^{-1}$ at 12 microns and $\sim 1.7$ MJy ster $^{-1}$ at 25 microns (Reach et al. 1995). We searched the DIRBE data set for a similar wake of dust trailing Mars.

Blackbody dust at the heliocentric distance of Mars has a typical temperature of $\sim 220 \mathrm{~K}$; it emits most strongly in the 12 and 25 micron DIRBE bands. We restricted our exploration to data from these two bands. We began by assembling a composite map of the emission from beyond the solar system, mostly due to stars and dust in the Galactic plane, by averaging together all the zodi-subtracted weekly maps in their native COBE quadrilateralized spherical cube coordinates, a coordinate system that is stationary on the celestial sphere. We subtracted this composite map from each of the zodi-subtracted weekly maps, effectively removing most of the galactic emission and any other stationary emission except within a few degrees of the galactic plane, where the emission is so high that detector and pointing instabilities make our linear subtraction method ineffective.

The remaining maps, with outlying data removed, had surface brightness residuals in the range of -1.7 to $+1.0 \mathrm{MJy} \mathrm{ster}^{-1}$ at 12 microns, and -1.6 to $2.1 \mathrm{MJy}^{\mathrm{ster}}{ }^{-1}$ at 25 microns. For comparison, the typical total zodiacal background near Mars during the mission is $\sim 35 \mathrm{MJy} \mathrm{ster}^{-1}$ at 12 microns and $\sim 66 \mathrm{MJy}^{-1}$ ster $^{-1}$ at 25 microns. The most prominent remaining features were the stripes parallel to the ecliptic plane within a few degrees of the ecliptic plane that are associated with the asteroidal dust bands. The next most prominent remaining features were wide bands extending \pm 30 degrees from the ecliptic that appeared to follow the sun. The $12-25$ micron color temperature of the wide bands was $\sim 280 \mathrm{~K}$; they are probably residuals resulting from imperfect subtraction of the Earth's ring and wake. We assembled a crude map of the residual near-Earth flux by averaging together the galaxy-subtracted maps in geocentric ecliptic coordinates referenced to the position of the Sun. Subtracting this from the weekly maps cancelled most of the signal in the wide bands. Mars moved $87^{\circ}$ with respect to the Sun during the mission, allowing us to subtract this composite map without subtracting a significant flux from a wake moving with Mars. Figure 3a shows our map of the galactic background; Figure 3b shows the near-Earth residuals.

Next we chose subframes of each weekly map centered on the ecliptic coordinates of Mars in the middle of the week, and inspected them visually. No structure in the data 
appeared to move with Mars from week to week.

In order to understand the data better, we constructed a simple model of the Mars wake from the empirical model of the Earth's trailing wake fit to the DIRBE data by Kelsall et al. (1998). The model has the following form:

$$
n=n_{0} \exp \left[-\frac{\left(r-r_{0}\right)^{2}}{2 \sigma_{r}^{2}}-\frac{|z|}{\sigma_{z}}-\frac{\left(\theta-\theta_{0}\right)^{2}}{2 \sigma_{\theta}^{2}}\right]
$$

where $n$ is the local average of particle number density times particle cross section, and $r, z$, and $\theta$ are cylindrical coordinates in the plane of the orbit of Mars centered on the sun. Mars is located at $r=r_{0}, z=0, \theta=0$. The parameters of the model, $\theta_{0}, \sigma_{r}, \sigma_{z}, \sigma_{\theta}$, and $n_{0}$, are the same as the corresponding parameters for the Earth's wake: $\theta_{0}=-10^{\circ}, \sigma_{r}=0.10$ $\mathrm{AU}, \sigma_{z}=0.091 \mathrm{AU}, \sigma_{\theta}=12.1^{\circ}$. The shaded area trailing Mars in Figure 1 shows how this model would appear viewed from above the ecliptic plane. The Kelsall et al. (1998) Earth wake has $n_{0}=1.9 \times 10^{-8} \mathrm{AU}^{-1}$, but we chose $n_{0}=1.08 \times 10^{-8} \mathrm{AU}^{-1}$ so that the density of the model would be proportional to the local background dust density at the orbit of Mars. The model represents what the Earth wake would look like if it were trailing Mars instead of Earth.

We evaluated the model's surface brightness by computing the line-of-sight integral

$$
I_{\lambda}=E_{\lambda} \int n(r, z, \theta) B_{\lambda}(T) d s
$$

where $B_{\lambda}(T)$ is the Planck function and $E_{\lambda}$ is an emissivity modification factor prescribed by the COBE model to account for the deviation of the Earth wake's spectrum from a blackbody; $E_{12 \mu \mathrm{m}}=1.06, E_{25 \mu \mathrm{m}}=1.00$. The temperature of the dust varies with heliocentric distance, $R$, as $T=286 \mathrm{~K} R^{-0.467}$, following the DIRBE model. This expression is similar to what you would expect for grey-body dust $\left(T=278 \mathrm{~K}^{-0.5}\right)$.

In Figure 4, we compare a synthesized image of the model wake with a backgroundsubtracted image of the infrared sky around Mars. The image shows the flux in the 25 micron band averaged over weeks 26-34 (14 May 1990 to 15 July 1990) in ecliptic coordinates referenced to the position of Mars. The subset of the weekly 25-micron data used in this image is indicated by the horizontal stripes labeled "M" in Figure 2. Mars moved 40 degrees in ecliptic longitude over this period. The DIRBE team blanked the data within a square about $2.5^{\circ}$ on a side centered on Mars, and within a $1.5^{\circ}$ radius circle centered on Jupiter. A software mask in Figure 4 covers the region around Mars affected by this processing. This whole region, up to $40^{\circ}$ behind Mars, shows no sign of a brightness enhancement that we would associate with a wake of dust trailing Mars.

Maps from the later weeks suffer from an oversubtraction due to imperfections in the Kelsall et al. (1998) zodi model, visible as the dark region to the lower right. Weeks earlier 
in the mission suffer from a similar undersubtraction. These artifacts, our primary source of noise, appear to arise from dust bands at latitudes of $\sim \pm 10^{\circ}$, where bands associated with the Eos asteroids are prominent in the raw DIRBE data (Reach et al. 1997). As the dust from the asteroid belt spirals towards the sun, perturbations from planets deform the bands. The Kelsall et al. model includes a simple model of this dust band which could not take these perturbations into account. We chose the span of weeks used to create Figure 4 to minimize these artifacts, which are easily discernible by their extent in latitude and longitude.

To better compare the model with the data, we focused on a narrow strip with a height of $3^{\circ}$ in ecliptic latitude, extending from $8^{\circ}$ ahead of Mars to $39^{\circ}$ behind Mars in ecliptic longitude. This strip contains most of the flux in the model wake. We averaged together maps from weeks 26-34 prepared as described above to produce an image of this strip. In Figure 5, we plot a cut through this strip, and we compare it with the model, processed in the same manner as the data. The data are dominated by residuals from the ecliptic bands and the Earth's ring, smeared out in the ecliptic plane by the orbital motion of Mars. The standard deviation of the data is $0.54 \mathrm{MJy} \mathrm{ster}^{-1}$; although the distribution of the residuals is not Gaussian, based on this comparison we can place a rough $3-\sigma$ upper limit on the central peak of the Mars wake of $18 \%$ of the flux expected from our simple model.

The empirical model of the Earth's wake we have used for comparison to the Mars dust environment is not an ideal model for the Mars wake. It may not even be a good representation of the Earth's wake. Since COBE viewed the Earth wake from near the Earth only, the observations constrain the product $n_{0} \sigma_{\theta}$ for the Earth wake, but do not provide good constraints on either of these parameters alone. Kelsall et al. (1998) quote a formal error of $28 \%$ on the determination of $\sigma_{\theta}$. Calculations for 12 micron particles suggest that $\sigma_{\theta}$ for the Earth wake might be $40 \%$ lower than the Kelsall et al. (1988) number; this figure is based on Figure 5 in Dermott et al. (1994). Since we are sensitive to the wake's surface brightness peak as seen from the Earth, not Mars, using a more compact wake model affects our upper limits. Holding $n_{0} \sigma_{\theta}$ constant and decreasing $\sigma_{\theta}$ by $40 \%$ translates into a decrease of our upper limit to $11 \%$ of one Earth wake.

Mars has $11 \%$ of the mass of the Earth, so we expect it to trap less dust than the Earth, but not simply $11 \%$ as much dust. In fact, there is no simple scaling law that describes how the density of a dust ring relates to the size of the planet that traps it. The density of the Mars ring is proportional to the capture probability times the trapping time for each resonance summed over all relevant resonances and the distribution of particle sizes. In the adiabatic theory for resonant capture due to Poynting-Robertson drag, the capture probabilities depend on the mass of the planet compared to the mass of the star 
and on the eccentricity of the particle near resonance and (Beauge and Ferraz-Mello 1994). So one complicating factor is that the orbits of the dust particles are slightly more eccentric when they pass Mars than when they pass the Earth; a particle released on the orbit of a typical asteroid, at $2.7 \mathrm{AU}$ with an eccentricity of 0.14 , will have an eccentricity of 0.07 as it passes Mars, and an eccentricity of 0.04 when it passes the Earth (Wyatt \& Whipple 1950). The higher eccentricity makes them harder to trap.

The trapping time scale is proportional to the time it takes for the resonant interaction to significantly affect the eccentricity and libration amplitude of the particle. When the planet has a circular orbit, these time scales are on the order of the local Poynting-Robertson decay time (Liou and Zook 1997), which scales as $r_{0}^{2} / \beta$, where $\beta$ is the ratio of the Sun's radiation-pressure force on a particle to the Sun's gravitational force on the particle. Compared to the P-R drag at the heliocentric distance of the Earth, the Poynting-Robertson drag force at the orbit of Mars is less for a given particle by a factor of $1.52^{2}=2.31$. The small mass of Mars and the higher eccentricities of the orbits of the incoming particles work against the formation of a dense ring, but the greater heliocentric distance of Mars compared to the Earth works in favor of the formation of the ring.

So far our discussion has assumed that the trapping is adiabatic - that the orbital elements of the particles change on time scales much longer than the orbital period. This approximation may not be as good for trapping by Mars as it is for trapping by the Earth. Mars has a greater orbital eccentricity $(e=0.093)$ than the Earth $(e=0.017)$. This increases the widths of the zones of resonance overlap, and makes a larger fraction of dust orbits chaotic (Murray and Holman 1997).

Predicting the density of the Mars wake is another step more complex than predicting the density of the Mars ring. Compared to the Earth wake, the Mars wake may form closer to the planet and have a smaller $\sigma_{\theta}$. Since Mars is less massive than the Earth, a given particle would need to have a closer interaction with Mars than with the Earth to receive an impulse from the planet's gravity that would balance the Poynting-Robertson drag on the particle (Weidenschilling and Jackson 1993). For this reason, we expect the trapped particles which form the Mars wake to prefer resonant orbits with higher $j$ and lower $\phi$ than similar particles trapped by the Earth, where $\phi$ is the angle between the perihelion of the orbit of a particle and the longitude of conjunction of the particle and the planet. Our upper limit shows that the Mars wake is less dense than the Earth wake by more than the simple factor of the mass ratio times the square of the ratio of the semimajor axes $=0.11 \times 2.31=0.25$. However, a thorough numerical simulation which includes the effects we mentioned and others such as resonant interactions with Jupiter may be the only good way to relate our upper limit to the dynamical properties of the dust near Mars. 


\section{Trojan Dust}

While the Earth and Mars can collect abundant low eccentricity particles from all different orbital phases spiraling in from the asteroid belt, Jupiter orbits in a distinctly different dust environment. Outside the asteroid belt, the dust background probably consists mainly of small particles with high orbital eccentricities: submicron particles released by asteroids or comets that are kicked by radiation pressure into more eccentric orbits than their parent bodies (Berg and Grün 1973; Mann and Grün 1995). There is also a stream of submicron particles from the interstellar medium (Grün et al. 1994, Grogan et al. 1996) and there are probably a few particles near Jupiter that originated in the Kuiper belt (Liou et al. 1996). Jupiter probably traps many of the small particles in 1:1 mean motion resonances (Liou and Zook 1995). However these small trapped particles should occupy both "tadpole" and "horseshoe" orbits, without a strong preference for either, and the locations of their Lagrange points vary with $\beta$ (Murray 1994). They probably form large, diffuse ring-like clouds which are difficult for us to detect.

But there is another potential source of dust that could form concentrated clouds we could hope to detect against the asteroid bands in the DIRBE data: the Trojan asteroids. This population of asteroids orbits the Sun at $\sim 5.2$ AU in 1:1 resonances with Jupiter, librating about Jupiter's L4 and L5 Lagrange points, roughly $60^{\circ}$ before and behind the planet. They number about as many as the main-belt asteroids.

Marzari et al. (1997) have simulated the collisional evolution of the Trojan asteroids, and concluded that collisions in the L4 swarm produce on the order of 2000 fragments in the 1-40 km diameter range every million years. If we simplisticly assume a equilibrium size distribution for the produced particles, $d n \propto a^{-3.5} d a$, where a is the particle radius (Dohnanyi 1969), we find that there are roughly $10^{23}$ particles in the 10-100 micron diameter size range produced every million years. These large particles are likely to stay in roughly the same orbits as their parent bodies, trapped by Jupiter in "tadpole" orbits - orbits that librate around a single Lagrange point. They could conceivably form detectable clouds at L4 and L5.

Liou and Zook (1995) calculated that 2-micron diameter particles will stay trapped in 1:1 resonances for $\sim 5000$ years. A 20-micron diameter particle at Jupiter's orbit experiences $1 / 10$ of the Poynting-Robertson acceleration of 2 micron particles, and will typically stay trapped for 10 times as long (Schuerman 1980). Assuming a trapping time of 5000 years $\times$ the dust grain diameter $/ 2$ microns, and emissivity appropriate for amorphous icy grains (eg. Backman and Paresce 1993), the 10-100 micron diameter particles in the Trojan cloud will emit a total flux, as viewed from the Earth, of $\sim 3 \times 10^{-4}$ MJy at 60 microns, a few orders of magnitude below our detection limit. 
However, this is a drastic extrapolation and probably a poor guess at the actual cloud brightness; the size-frequency distribution of the Trojan asteroids is not well known and dust cloud is probably not near collisional equilibrium. Moreover, the total amount of trapped dust is subject to severe transients, such as the events that produced the dust bands associated with main belt asteroid families (Sykes and Greenberg 1986). For example, a 20-km diameter Trojan asteroid ground entirely into 10-micron diameter dust corresponds to a transient cloud which, as viewed from the Earth, would produce a 60 micron flux of $\sim 6$ MJy. A similarly enhanced cloud might be visible a few percent of the time.

Unfortunately, Jupiter's Lagrange points do not move far with respect to the galactic background during the COBE mission; L4 moves 10 degrees and L5 moves 50 degrees, as shown in Figure 2. Only L5, the trailing Lagrange point, moves far enough during the mission to make subtracting the galactic background feasible. There are about half as many L5 Trojans known as L4 Trojans, but this is probably because the L5 region has been searched less intensely than the L4 region, not because the L4 and L5 populations are significantly different (Shoemaker et al. 1989).

To make a background-subtracted image of the L5 region, we chose two subsets from the zodi-subtracted data set. The first, subset A, is from the beginning of the mission (weeks 5-10) when L5 was in the viewing swath and approximately stationary on the sky. The second, subset B, is the same region of sky, but contains data from half a year later in the mission (weeks 33-38), when L5 has moved 45 degrees away, out of the viewing swath. The average distance from Earth to L5 is approximately the same during each time period. These data sets are depicted in Figure 2. We focused on data in the 60 micron band, the band which contains the emission peak for dust at the local blackbody temperature at 5.2 AU. To minimize the residuals from the zodiacal dust model, we used only data from solar elongations between 65 and 115 degrees, (or between 245 and 295 degrees). Figure 6 shows an image constructed from data set $\mathrm{A}$ and an image constructed from data set $\mathrm{B}$, and the difference, $\mathrm{A}-\mathrm{B}$, which is dominated by residuals from dust bands associated with asteroid bands and shows no obvious evidence of enhanced emission at L5.

We made a simple model for a Trojan cloud of large dust particles by assuming that they occupy the same dynamical space as the Trojan asteroids themselves, following Sykes (1990). Sykes modeled the asteroidal dust bands by showing how particles constrained to orbits with a given inclination, eccentricity, and semimajor axis form a cloud when the remaining three orbital elements are randomized. He then convolved the shapes of these clouds with the distributions of orbital elements of the asteroids. In our case, however, the distribution of orbital elements is much broader and more important in determining the shape of the final distribution of particles. Since there are only about 70 Trojan asteroids 
whose orbits are well studied, the distributions of Trojan asteroid orbital parameters have severe statistical uncertainties. Therefore we settle for a simple Gaussian model for the Trojan cloud, using the orbital parameters as a guide to the parameters of the Gaussian. In the following calculations, we will neglect the inclination of Jupiter's orbit relative to the the Earth's orbit $\left(1.305^{\circ}\right)$.

A typical Trojan asteroid librates around its Lagrange point with a period of 148 days. The mean longitude of the asteroid with respect to Jupiter, $\phi$, oscillates within limits $\phi_{\min }$ and $\phi_{\max }$, which can be calculated, according to Yoder et al. (1983), from

$$
\sin \frac{\phi_{\min }}{2}=\frac{\sin (\alpha / 3)}{B} \quad \sin \frac{\phi_{\max }}{2}=\frac{\sin \left(\alpha / 3+120^{\circ}\right)}{B}
$$

where $B=\eta_{0}(3 \mu / 2 E)^{1 / 2}, \sin \alpha=B, \mu=\mathrm{M}_{\text {Jupiter }} / \mathrm{M}_{\odot}=0.000955$ and $\eta_{0}=$ mean motion of Jupiter $=0.01341 \mathrm{rad} \mathrm{yr}^{-1}$. These limits are set by $E$, which is a constant of the motion in the absence of Poynting Robertson drag:

$$
E=-\frac{1}{6}\left(\frac{d \phi}{d t}\right)^{2}-\frac{\mu \eta_{0}^{2}}{2 x}\left(1+4 x^{3}\right)
$$

where $x=|\sin (\phi / 2)|$. The libration amplitude, $D$, is $\phi_{\max }-\phi_{\min }$. We find that the energy constant is approximately

$$
E \approx-\frac{3}{2} \mu \eta_{0}^{2}\left(1-0.0133 D+0.2266 D^{2}-0.0392 D^{3}\right)
$$

for $D \leq 1.3$.

The fraction of time a particle spends at a given phase, or equivalently, the distribution in phase of an ensemble of particles is given by

$$
P_{\phi} \propto \frac{1}{d \phi / d t}
$$

We can evaluate this as a function of $D$ with the aid of equations (4) and (5). For the distribution of dust libration amplitudes, $P_{D}$, we used a simple analytic function that approximates the distribution of libration amplitudes for Trojan asteroids shown in Figure 5 of Shoemaker et al. (1989). When we average $P_{\phi}$ over $P_{D}$, we find that the L5 dust cloud is distributed in orbital phase roughly as a Gaussian centered at $\theta_{0}=59.5^{\circ}$ behind Jupiter with a dispersion $\sigma_{\theta}=10^{\circ}$.

The distribution of the dust in heliocentric latitude can be approximated in a similar way. If particle in an orbit of given inclination, $i$, with small eccentricity, spends a fraction 
of its time, $f$, at latitude, $\beta$, an ensemble of particles with small eccentricities and evenly distributed ascending nodes will have a distribution, at a fixed orbital phase, of

$$
P_{\beta} \propto f \propto\left(\cos ^{2} \beta-\cos ^{2} i\right)^{-1 / 2}
$$

We takes the inclination distribution of the particles, $P_{i}$, to be a simple analytic function that approximates the data for "independently discovered Trojans" shown in Figure 3 of Shoemaker et al. (1989). When we average $P_{\beta}$ over $P_{i}$, we find the distribution in latitude is roughly a Gaussian with dispersion $\sigma_{\beta}=10^{\circ}$, and the distribution in height above the ecliptic has a dispersion $\sigma_{z}=0.94 \mathrm{AU}$.

The radial distribution of Trojans is more complicated to model, since both librations and epicycles include radial excursions. The average L5 Trojan eccentricity is 0.063; a particle with this eccentricity orbits at a range of heliocentric distances, $\Delta r \approx 0.66 \mathrm{AU}$. In the course of its librations, a particle with a typical Trojan libration amplitude, $D=29^{\circ}$, oscillates in semi-major axis over a range of $\Delta a \approx 0.14 \mathrm{AU}$. We are not sensitive to the radial structure of the Trojan clouds, so we simply model the radial distribution as a Gaussian with a full width at half maximum of $0.66 \mathrm{AU}$, or a dispersion $\sigma_{r}=0.24 \mathrm{AU}$.

Our final model has the form:

$$
n=n_{0} \exp \left[-\frac{\left(r-r_{0}\right)^{2}}{2 \sigma_{r}^{2}}-\frac{z^{2}}{2 \sigma_{z}^{2}}-\frac{\left(\theta-\theta_{0}\right)^{2}}{2 \sigma_{\theta}^{2}}\right]
$$

where $r, z$, and $\theta$ are cylindrical coordinates in the plane of the orbit of Jupiter, and the parameters are: $r_{0}=5.203 \mathrm{AU}, \sigma_{r}=0.24 \mathrm{AU}, \sigma_{z}=0.94 \mathrm{AU}, \theta_{0}=-59.5^{\circ}$, and $\sigma_{\theta}=9.7^{\circ}$. We calculated the surface brightness in the same way as we calculated the surface brightness of the model Mars wake, using an emissivity $E_{60 \mu \mathrm{m}}=1$ because we are not considering small grains. The shaded region at L5 in Figure 1 represents this model as viewed from above the ecliptic plane.

In Figure 6, we compare the difference image $\mathrm{A}-\mathrm{B}$ to a synthesized image of our model cloud. For this image, $n_{0}$ is $3.4 \times 10^{-8} \mathrm{AU}^{-1}$, corresponding to an effective emitting surface area at 60 microns of $3.3 \times 10^{18} \mathrm{~cm}^{2}$, or one $3-\mathrm{km}$ diameter asteroid ground entirely into 10-micron diameter dust. Figure 7 compares the difference image A-B and the model image in a different way; it shows the region within $\pm 10^{\circ}$ of the ecliptic plane averaged in ecliptic latitude. The 1- $\sigma$ noise in the data in Figure 7 is $0.09 \mathrm{MJy}_{\text {ster }}{ }^{-1}$. Based on this, we can place a rough 3- $\sigma$ upper limit on the effective surface area of the large dust grains at L5 of $\sim 6 \times 10^{17} \mathrm{~cm}^{2}$. 


\section{Conclusions}

The zodiacal cloud near the ecliptic plane is a complex tapestry of dynamical phenomena. We could not detect the Mars wake or Jupiter's Trojan clouds among the asteroidal dust bands in the DIRBE maps, despite the efforts of the DIRBE team to subtract these bands from the maps. We would have detected the Mars wake if it had $18 \%$ of the overdensity of the Earth wake, based on our empirical model for the Earth wake. This upper limit illustrates the complexity of relating resonant structures in circumstellar dust disks to the properties of perturbing planets. For instance, we would have detected the Mars wake if the surface area of the dust in the wake scaled simply with the mass of the planet times the Poynting-Robertson time scale.

The Trojan clouds, by our crude estimation, would have been a few orders of magnitude too faint to detect if the dust concentration in these clouds were at its mean levels. However, a transient cloud created by a recent collision of Trojan asteroids might have been

detectable. We measured that the total 60-micron flux from large (10-100 micron diameter) dust particles trapped at Jupiter's L5 Lagrange point is less than $\sim 30 \mathrm{kJy}$.

We thank Antonin Bouchez, Eric Gaidos, Peter Goldreich, Renu Malhotra and Ingrid Mann for helpful discussions, and our referees for their thoughtful comments.

\section{REFERENCES}

Beaugé, C., and S. Ferraz-Mello 1994. Capture in Exterior Mean-Motion Resonances Due to Poynting-Robertson Drag. Icarus 110, 239-260

Beichman, C. A. 1998. Sensitivity of the Terrestrial Planet Finder. In Exozodiacal Dust Workshop Conference Proceedings (D. E. Backman, L. J. Caroff, S. A. Sandford, and D. H. Wooden, Eds.), pp. 149-172 (NASA/CP-1998-10155)

Beichman, C. A., Woolf, N. J., \& Lindensmith, C. A. (eds) 1999, JPL Publication 99-3, The Terrestrial Planet Finder (Pasadena: JPL)

Berg, O. E., and E. Grün 1973. Evidence of Hyperbolic Cosmic Dust Particles. In Space Research XIII, p. 1047 Akademie-Verlag, Berlin.

Boggess, N. W., Msther, J. C., Weiss, R., Bennett, C. L., Cheng, E. S., Dwek, E., Gulkis, S., Hauser, M. G., Janssen, M. A., Kelsall, T., Meyer, S. S., Moseley, S. H., Murdock, T. L., Shafer, R. A., Silverberg, R. F., Smoot, G. F., Wilkinson, D. T., Wright, E. L. 1992, ApJ, 379, 420 
COBE Diffuse Infrared Background Experiment (DIRBE) Explanatory Supplement, ed. M.G. Hauser, T. Kelsall, D. Lesiawitz, and J. Weiland, COBE Ref. Pub. No. 97-A (Greenbelt, MD: NASA/GSFC), available in electronic form from the NSSDC

Dermott, S.F., S. Jayaraman, Y. L. Xu, B.Å.S. Gustafson, and J. C. Liou 1994. A Circumsolar Ring of Asteroidal Dust in Resonant Lock With The Earth. Nature 369, 719-723

Dermott, S.F., P. D. Nicholson, Y. Kim, B. Wolven, and E. F. Tedesco 1988. The Impact of IRAS on Asteroidal Science. In Comets to Cosmology (A. Lawrence, Ed.), pp. 3-18. Springer-Verlag, Berlin

Grogan, K., S. F. Dermott, and B. Å. S. Gustafson 1996. Astrophys. J. 472, 812-817

Grün, E., B. Å. S. Gustafson, I. Mann, M. Baguhl, G. E. Morfill, P. Staubach, A. Taylor, and H. A. Zook 1994. Astron. Astrophys. 286, 915-924

Jackson, A. A., and H. A. Zook 1989. A Solar System Dust Ring with the Earth as its Shepherd. Nature 337, 629-31

Kelsall, T., J. L. Weiland, B. A. Franz, W. T. Reach, R. G. Arendt, E. Dwek, H. T. Freudenreich, M. G. Hauser, S. H. Moseley, N. P. Odegard, R. F. Silverberg, R. F. and E. L. Wright 1998. The COBE Diffuse Infrared Background Experiment Search for the Cosmic Infrared Background. II. Model of the Interplanetary Dust Cloud. Astrophys. J. 508, 44-73

Liou, J.C., and H. A. Zook 1995. An Asteroidal Dust Ring of Micron Sized Particles Trapped in the 1:1 Mean Motion Resonance with Jupiter. Icarus 113, 403-414

Liou, J. C., and H. A. Zook 1997. Evolution of Interplanetary Dust Particles in Mean Motion Resonances. Icarus, 128, 354-367

Liou, J.C., H. A. Zook, and S. F. Dermott 1996. Kuiper Belt Dust Grains as a Source of Interplanetary Dust Particles. Icarus 124, 429-440

Mann, I., and E. Grün 1995. Dust Particles Beyond The Asteroid Belt-a Study Based on Recent Results of the Ulysses Dust Experiment. Planet. Space Sci. 43, 827-832

Marzari, F., P. Farinella, D. R. Davis, H. Scholl, and A. Campo Bagatin 1997. Collisional Evolution of Trojan Asteroids. Icarus 125, 39-49

MacPhie, R. H. and R. N. Bracewell 1979. An Orbiting Infrared Interferometer to Search for Non-Solar Planets. Proc. SPIE 172, 271-278

Murray, C. Dynamical Effects of Drag in the Circular Restricted Three-Body Problem. Icarus. $112,465-484$ 
Murray, N., and M. Holman 1997. Diffusive Chaos in the Outer Asteroid Belt. Astron. J. $114,1246-1259$

Reach, W.T. 1991. Zodiacal Emission. II. Dust Near Ecliptic Astrophys. J. 369, 529-543

Reach, W.T., B. A. Franz, J. L. Weiland, M. G. Hauser, T. N. Kelsall, E. L. Wright, G. Rawley, S. W. Stemwedel, and W. J. Spiesman 1995. Observational Confirmation of a Circumsolar Dust Ring by the COBE Satellite. Nature 374, 521-523

Reach, W.T., B. A. Franz, and J. L. Weiland 1997. The Three Dimensional Structure of the Zodiacal Dust Bands. Icarus 127, 461-484

Roques, F., H. Scholl, B. Sicardy, and B. A. Smith 1994. Is There a Planet around Beta Pictoris? Perturbations of a Planet on a Circumstellar Dust Dist: 1 The Numerical Model. Icarus 108, 37-58

Schuerman, D. W. 1980. The Restricted Three-Body Problem Including Radiation Pressure. Astrophys. J. 238, 337-342

Shoemaker, E. M., C. S. Shoemaker, and R. F. Wolfe 1989. Trojan Asteroids: Populations, Dynamical Structure and Origin of the L4 and L5 Swarms. In Asteroids II (R. P. Binzel, T. Gehrels, and M. S. Matthews, Eds.), pp. 487-523. Univ. of Arizona Press, Tucson.

Spiesman, W. J., M. G. Hauser, T. Kelsall, C. M. Lisse, S. H. Moseley, W. T. Reach, R. F. Silverberg, S. W. Stemwedel, and J. L. Wieland 1995. Near- and Far-Infrared Observations in Interplanetary Dust Bands From the COBE Diffuse Infrared Background Experiment. Astrophys. J. 442, 662-667

Sykes, M. V. 1990. Zodiacal Dust Bands: Their Relation to Asteroid Families. Icarus 84, 267-289

Sykes, M. V. and R. Greenberg 1986. The Formation and Origin of the IRAS Zodiacal Dust Bands as a Consequence of Single Collisions between Asteroids. Icarus 65, 51-69

Weidenschilling, S. J., and A. A. Jackson 1993. Orbital Resonances and Poynting-Robertson Drag. Icarus 104, 244-254

Wyatt, M. C., S. F. Dermott, C. M. Telesco, R. S. Fisher, K. Grogan, E. K. Holmes, and R. K. Piña 1999. How Observations of Circumstellar Disk Asymmetries Can Reveal Hidden Planets: Pericenter Glow and its Application to the HR 4796 Disk. In preparation.

Wyatt, S. P. and F. L. Whipple 1950. The Poynting-Robertson Effect On Meteor Orbits. Astrophys. J. 111, 134-141 
Yoder, C.F., G. Colombo, S. P. Synnott, and K. A. Yoder 1983. Theory of Motion of Saturn's Coorbiting Satellites. Icarus 53, 431-443

This preprint was prepared with the AAS LATEX macros v4.0. 
Fig. 1. - The solar system during week 34. The shaded regions following Mars represents our model for the Mars wake; the shaded region centered on L5 represents our model for the Trojan cloud. The hatched area represents the DIRBE viewing swath for that week.

Fig. 2.- The ecliptic longitudes of the Sun, Mars, Jupiter, and Jupiter's L4 and L5 Lagrange points during 40 weeks of the COBE mission when DIRBE was recording. The shaded diagonal stripes show the intersection of the DIRBE viewing swath with the ecliptic plane. The vertical dashed lines show where the galactic plane crosses the ecliptic. The horizontal bars show the data sets used to construct Figures 4,5,and 6 .

Fig. 3.- Two 25-micron backgrounds that we subtracted from the Mars images. a) The galactic background, constructed by averaging all the weekly maps in their native quadrilateralized spherical cube coordinates. b) Residuals from the Earth's wake, constructed by averaging all the weekly galaxy-subtracted maps in a geocentric ecliptic coordinate system with the sun at the origin.

Fig. 4.- An image of the sky near Mars at 25 microns, compared to a model based on the COBE DIRBE empirical model for the wake trailing Earth. The image is averaged over weeks 26-34 (data set M). The region within $1.5^{\circ}$ of Mars has been covered by a software mask.

Fig. 5.- A cut through the image of the 25-micron sky near Mars shown in Figure 4, compared to the same model.

Fig. 6.- Raw DSZA images in the ecliptic plane at 60 microns. L5 is at the center of image $\mathrm{A}$, but it has moved 45 degrees to the right of center in image $\mathrm{B}$. The difference, $\mathrm{A}-\mathrm{B}$ cancels most of the galactic emission, but is dominated by residuals from dust bands associated with the asteroid belt and does not reveal any Trojan dust. The model shows what we would expect the difference $\mathrm{A}-\mathrm{B}$ to look like, given some simple assumptions about the Trojan clouds.

Fig. 7.- The difference A-B compared to the model for the L5 cloud. This plot shows a region of the 60 -micron maps from Figure 6 within $\pm 10^{\circ}$ of the ecliptic plane that has been averaged in latitude. Based on this comparison, we place a 3- $\sigma$ upper limit on the surface area of the L5 cloud of $6 \times 10^{17} \mathrm{~cm}^{2}$. 


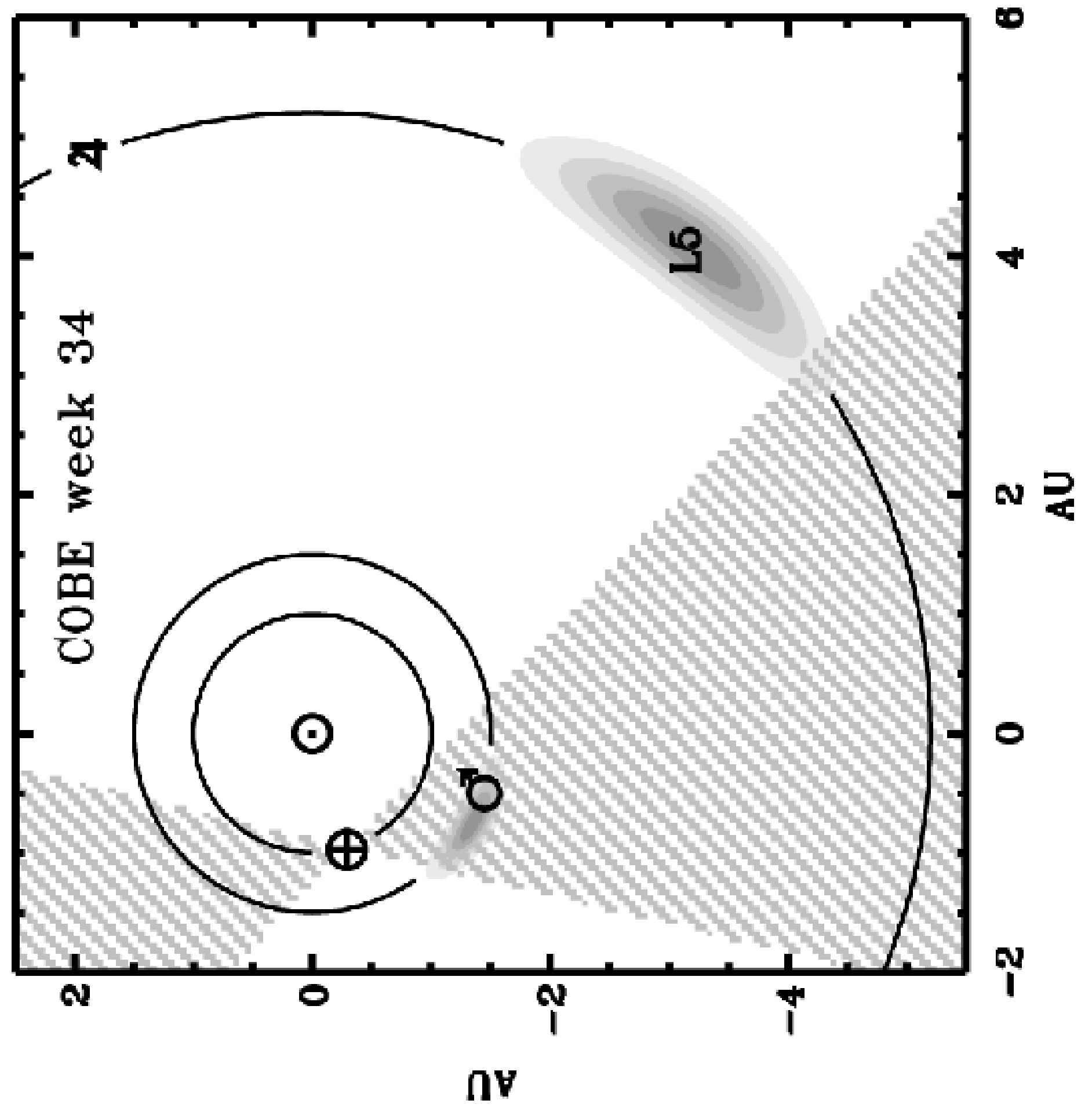




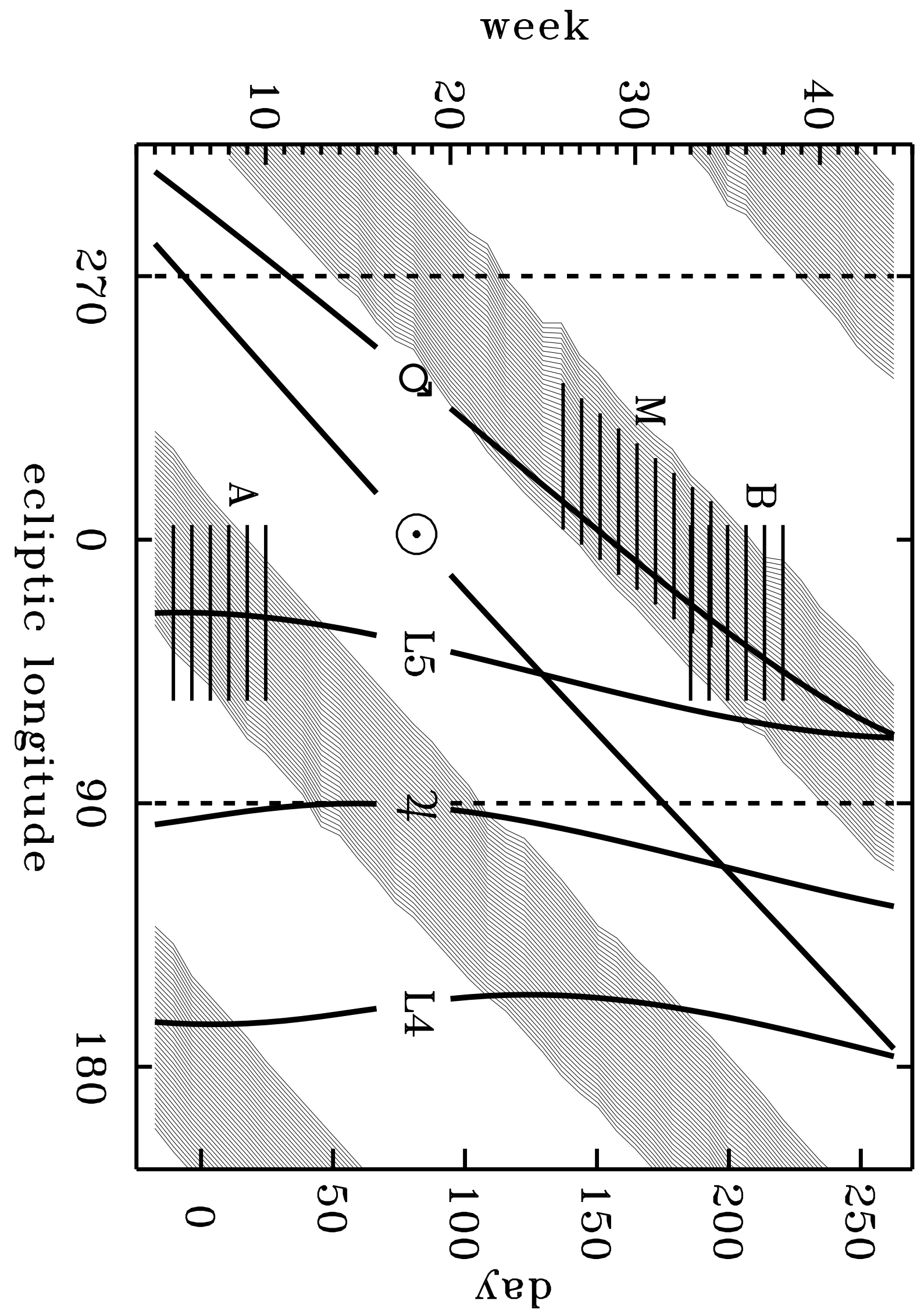


This figure "figure3a.gif" is available in "gif" format from: http://arxiv.org/ps/astro-ph/0002038v1 
This figure "figure3b.gif" is available in "gif" format from: http://arxiv.org/ps/astro-ph/0002038v1 


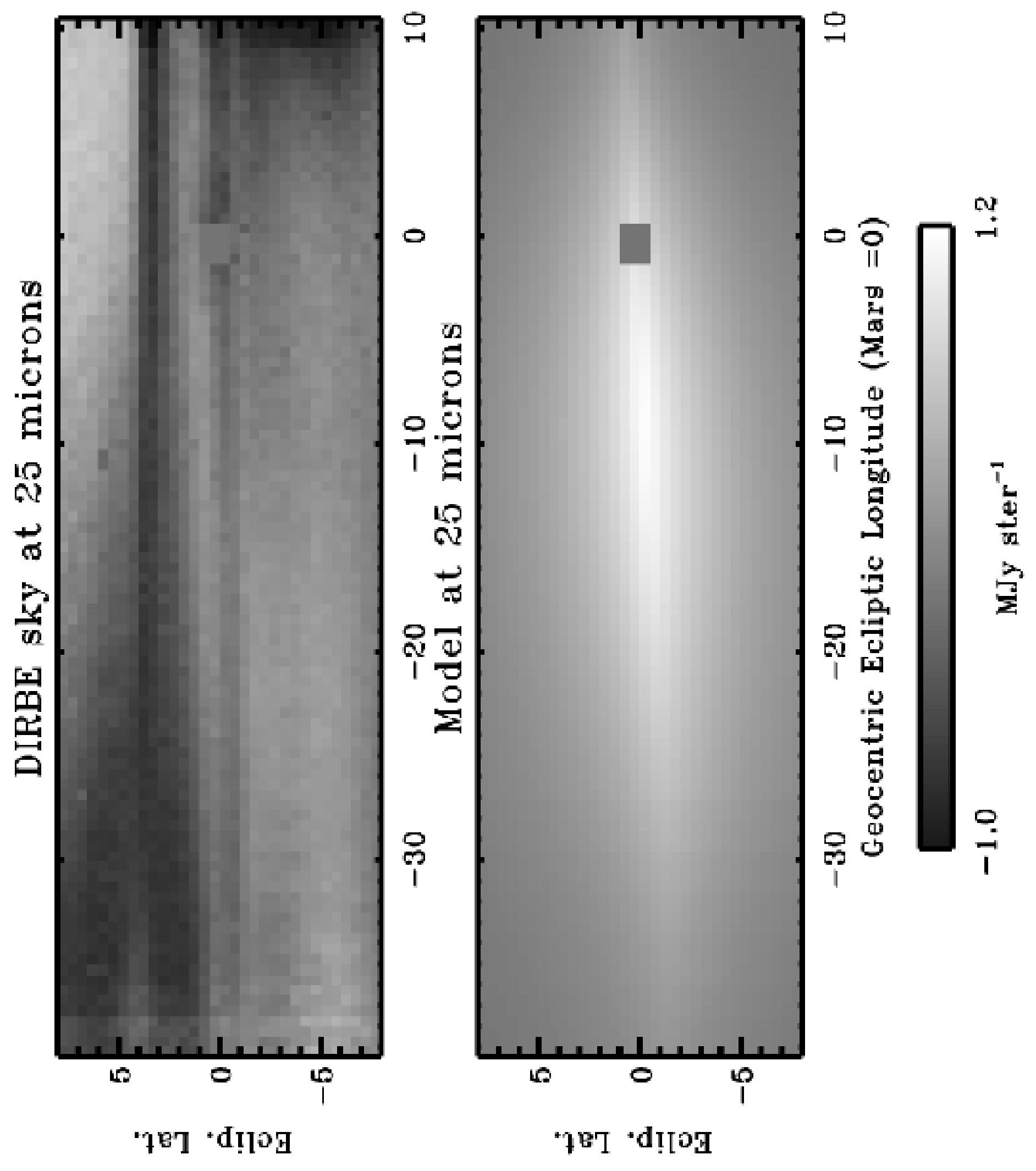




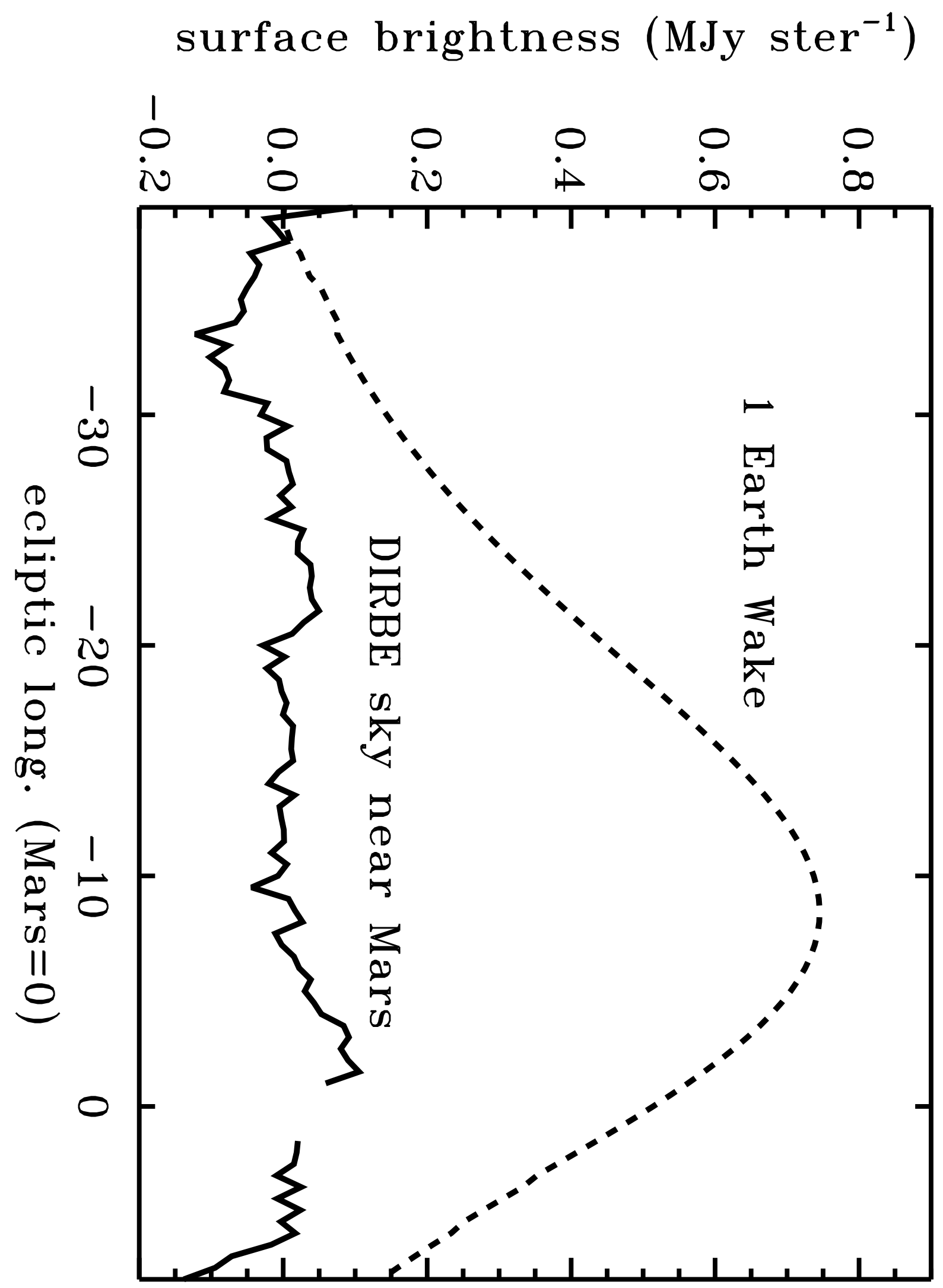




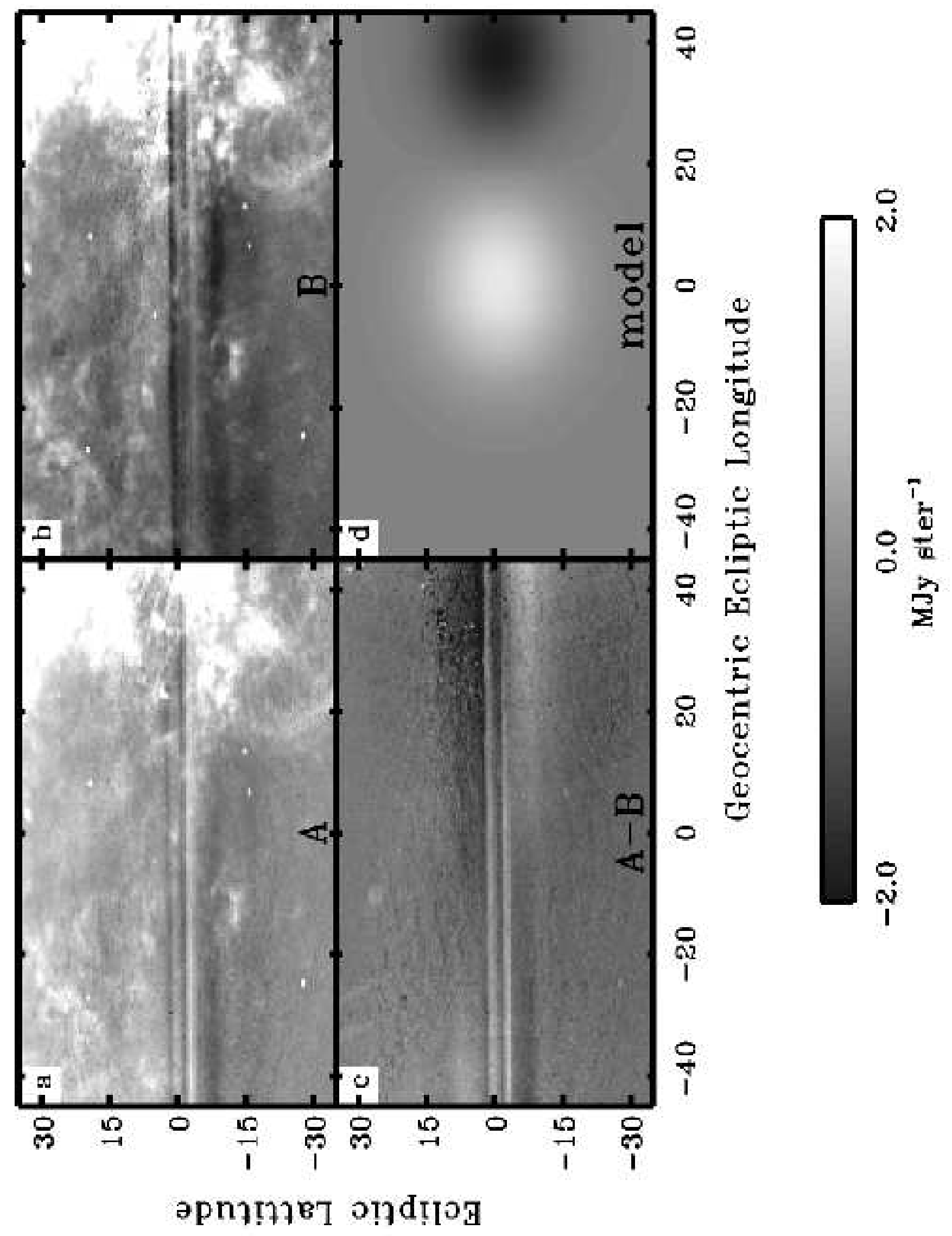




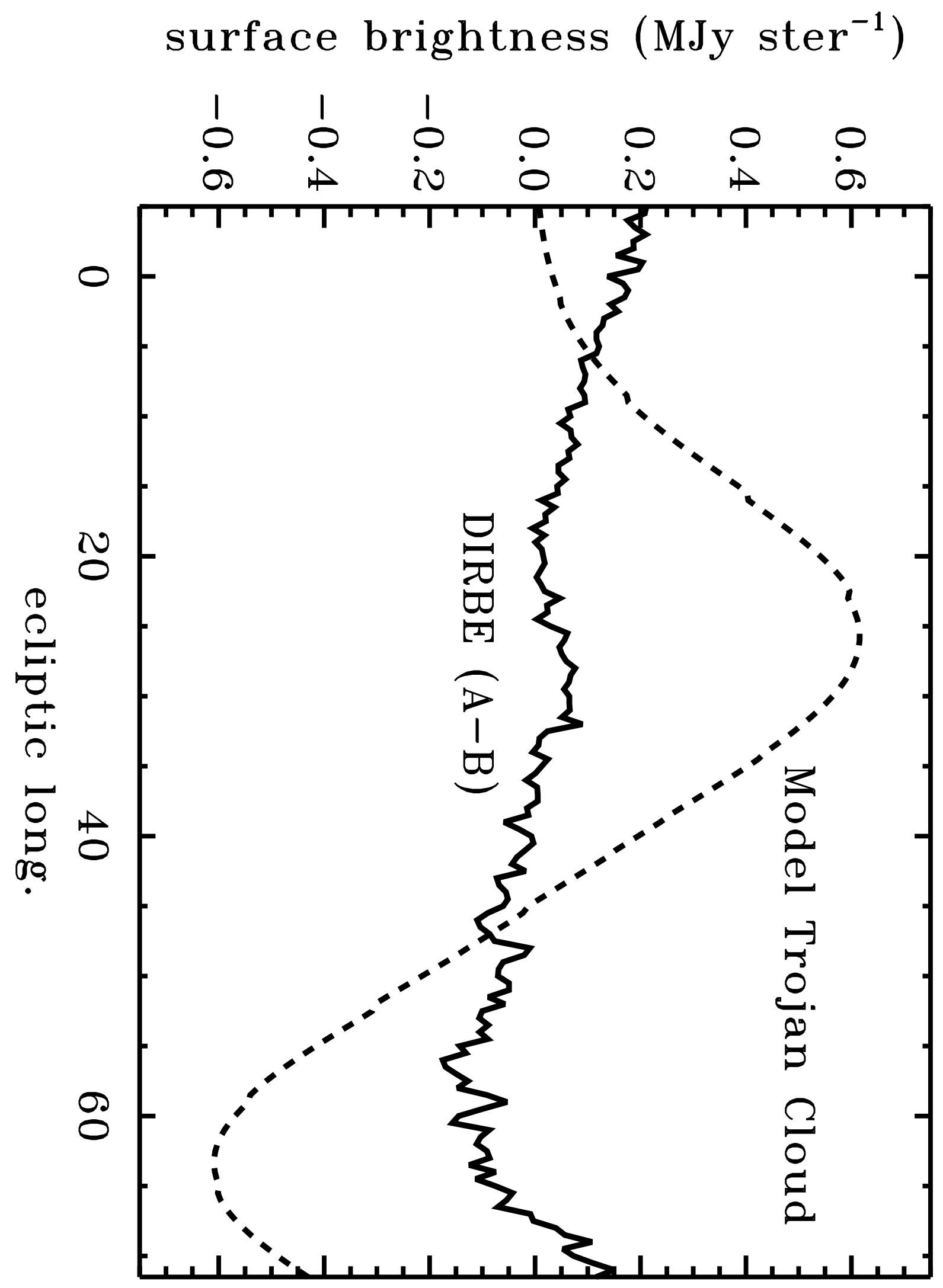

